\title{
Non-linear shape oscillations of a viscous liquid drop
}

\author{
Dino Zrnić, Günter Brenn \\ Institute of Fluid Mechanics and Heat Transfer, Graz University of Technology, Graz, Austria \\ *Corresponding author email: guenter.brenn@tugraz.at
}

\begin{abstract}
Non-linear oscillations of a viscous Newtonian liquid drop are investigated for fundamental interest and for their relevance for transport processes across the drop surface. Theoretically we adopt the weakly non-linear approach to account for the influence of the non-linear motion on the oscillations. Non-linearities gain importance by strong drop deformation and depend on the initial internal flow. Experiments show a different behaviour for large and for small oscillation amplitudes, seen weakly in the oscillation frequency, but strongly in the damping rate.
\end{abstract}

\section{Keywords}

Drop shape oscillations; weakly non-linear analysis; angular oscillation frequency; excess time.

\section{Introduction}

Shape oscillations of drops have been studied in science since more than 140 years. Drop shape oscillations are of interest, since they influence both the transport of heat and mass across the drop surface, and the aerodynamic drag of the drop. In the appendix to his paper on the capillary phenomena of jets, Rayleigh presented an analysis of linear shape oscillations of an inviscid drop in a vacuum around a spherical equilibrium state [1]. One result is the equation for the angular oscillation frequency of the drop deformed according to a mode $m$ counting the lobes along the drop surface. Lamb generalised Rayleigh's result by accounting for the drop viscosity and for the density of the ambient medium [2,3]. The threshold Ohnesorge number $O h=\mu /(\sigma a \rho)^{1 / 2}$ of the drop for the onset of aperiodic behaviour is predicted. A further generalisation of the analysis of linear drop shape oscillations was achieved in [4] by account for both the viscous and the inertial influences from the ambient medium hosting the viscous oscillating drop. The important aspect of the initiation of the drop oscillations in a drop initially at rest was analysed in [5], treating the motion as an initial-value problem. It was shown that the analyses of linear drop oscillations by the normal-mode approach may miss the fact that, in a range of Ohnesorge numbers, oscillations starting aperiodically may turn into periodic with ongoing time. The most important results of the research are the angular frequency and damping rate of the oscillation, as well as the shapes of the deformed drops in linear motion. Non-linear effects reveal a dependency of the oscillation frequency on the initial drop deformation from the spherical state. Tsamopoulos \& Brown were the first to investigate this for inviscid drops and bubbles [6]. They found shapes deviating from the linear results, and angular frequencies decreasing with increasing oscillation amplitude, for a maximum prolate aspect ratio of 1.4 by approximately $5 \%$. The sinusoidal dependency of the shape on time is lost with increasing oscillation amplitude, but still the predicted motion remained periodic.

The aim of the present work is to investigate non-linear viscous drop shape oscillations by the weakly non-linear method of analysis up to second order. The interest focuses on the dampening of viscous drop oscillations due to non-linear viscous effects. This advances the recent analysis for the inviscid drop [7].

\section{Theory - formulation of the problem}

We study the weakly non-linear shape oscillations of a viscous liquid drop.The drop is assumed to be axisymmetric with respect to the coordinate $\varphi$ of the spherical coordinate system. The liquid is treated as incompressible and Newtonian. Dynamic influences from an ambient medium 
is neglected, i.e. the drop is placed in a vacuum. Body forces are not accounted for. The problem is formulated in spherical coordinates to account for its geometry.

The variables and equations of change are non-dimensionalized with the undeformed drop radius $a$, the capillary time scale $\left(\rho a^{3} / \sigma\right)^{1 / 2}$ and the capillary pressure $\sigma / a$, where $\rho$ is the liquid density and $\sigma$ the liquid interfacial tension against the vacuum. The drop surface is described by its radial extension $r_{s}(\theta, t)=1+\eta(\theta, t)$, with the non-dimensional deformation $\eta$ against the undisturbed spherical shape.

For the problem at hand, the equation of continuity and the two components of the momentum equation in the radial $(r)$ and polar angular $(\theta)$ directions read

$$
\begin{aligned}
\frac{1}{r^{2}} \frac{\partial}{\partial r}\left(r^{2} u_{r}\right) & +\frac{1}{r \sin \theta} \frac{\partial}{\partial \theta}\left(u_{\theta} \sin \theta\right)=0 \\
\frac{\partial u_{r}}{\partial t} & +u_{r} \frac{\partial u_{r}}{\partial r}+\frac{u_{\theta}}{r} \frac{\partial u_{r}}{\partial \theta}-\frac{u_{\theta}^{2}}{r}=-\frac{\partial p}{\partial r} \\
& +O h\left[\frac{1}{r^{2}} \frac{\partial^{2}}{\partial r^{2}}\left(r^{2} u_{r}\right)+\frac{1}{r^{2} \sin \theta} \frac{\partial}{\partial \theta}\left(\frac{\partial u_{r}}{\partial \theta} \sin \theta\right)\right] \\
\frac{\partial u_{\theta}}{\partial t} & +u_{r} \frac{\partial u_{\theta}}{\partial r}+\frac{u_{\theta}}{r} \frac{\partial u_{\theta}}{\partial \theta}+\frac{u_{r} u_{\theta}}{r}=-\frac{1}{r} \frac{\partial p}{\partial \theta}+ \\
& +O h\left[\frac{1}{r^{2}} \frac{\partial}{\partial r}\left(r^{2} \frac{\partial u_{\theta}}{\partial r}\right)+\frac{1}{r^{2}} \frac{\partial}{\partial \theta}\left(\frac{1}{\sin \theta} \frac{\partial}{\partial \theta}\left(u_{\theta} \sin \theta\right)\right)+\frac{2}{r^{2}} \frac{\partial u_{r}}{\partial \theta}\right]
\end{aligned}
$$

where $O h=\mu /(\sigma a \rho)^{1 / 2}$ is the Ohnesorge number, with the liquid dynamic viscosity $\mu$. The solution of the above set of equations is subject to the kinematic boundary condition

$$
u_{r}=\frac{D \eta}{D t}=\frac{\partial \eta}{\partial t}+\frac{u_{\theta}}{r} \frac{\partial \eta}{\partial \theta} \quad \text { at } r=1+\eta,
$$

and the zero-shear stress boundary condition

$$
(\vec{n} \cdot \tau) \times \vec{n}=\overrightarrow{0} \quad \text { at } r=1+\eta
$$

where the outward unit normal vector $\vec{n}$ is given as

$$
\vec{n}=\frac{1}{|\vec{\nabla} F|} \vec{\nabla} F \quad \text { with } F=r-1-\eta(\theta, t)=0
$$

and the viscous extra stress tensor $\tau$. One further boundary condition states zero normal stress at the drop surface, i.e.,

$$
-p+O h(\vec{n} \cdot \tau) \cdot \vec{n}+(\vec{\nabla} \cdot \vec{n})=0 \quad \text { at } r=1+\eta
$$

We obtain the divergence of the normal unit vector in this equation as

$$
(\vec{\nabla} \cdot \vec{n})=\frac{1}{r} \frac{2+3\left(\frac{1}{r} \frac{\partial \eta}{\partial \theta}\right)^{2}}{\left[1+\left(\frac{1}{r} \frac{\partial \eta}{\partial \theta}\right)^{2}\right]^{3 / 2}}-\frac{1}{r^{2} \sin \theta} \frac{\partial}{\partial \theta}\left(\frac{\frac{\partial \eta}{\partial \theta}}{\left[1+\left(\frac{1}{r} \frac{\partial \eta}{\partial \theta}\right)^{2}\right]^{1 / 2}} \sin \theta\right) \quad \text { at } r=1+\eta
$$

Initial conditions state that the drop surface is initially deformed and at rest.

For analyzing these equations with the boundary and initial conditions in a weakly non-linear form, all the flow field properties are expanded in power series with respect to the deformation parameter $\eta_{0} \ll 1$. As an example,

$$
u_{r}=u_{r 1} \eta_{0}+u_{r 2} \eta_{0}^{2}+\ldots
$$


and the radial velocity component for use in the boundary conditions reads

$$
\left.u_{r}\right|_{r=1+\eta}=\left.u_{r}\right|_{r=1}+\left.\frac{\partial u_{r}}{\partial r}\right|_{r=1} \eta+\ldots
$$

Using this approach, the initial deformed drop shape, which is governed by a Legendre polynomial of degree $m$ and the amplitude $\eta_{0}$, reads

$$
r_{s}(\theta, 0)=1+\eta(\theta, 0)=1+\eta_{0} P_{m}(\cos \theta)-\eta_{0}^{2} \frac{1}{2 m+1}-\frac{\eta_{0}^{3}}{6} \int_{-1}^{1} P_{m}(\cos \theta)^{3} d(\cos \theta) \mp \ldots
$$

Substituting these approaches into the equations (1) - (3) and into the boundary conditions (4), (5) and (7), and representing the flow properties and their derivatives as given in (9) and (10), we obtain sets of first- and second-order equations of motion, with the boundary conditions, consisting of all the terms with the deformation parameter $\eta_{0}$ to the first and second powers, respectively.

\section{First-order equations}

The first-order equations consist of all the terms in the above expansions with the parameter $\eta_{0}$ to the first power. The first-order continuity and momentum equations read

$$
\begin{aligned}
\frac{1}{r^{2}} \frac{\partial}{\partial r}\left(r^{2} u_{r 1}\right) & +\frac{1}{r \sin \theta} \frac{\partial}{\partial \theta}\left(u_{\theta 1} \sin \theta\right)=0 \\
\frac{\partial u_{r 1}}{\partial t} & =-\frac{\partial p_{1}}{\partial r}+O h\left[\frac{1}{r^{2}} \frac{\partial^{2}}{\partial r^{2}}\left(r^{2} u_{r 1}\right)+\frac{1}{r^{2} \sin \theta} \frac{\partial}{\partial \theta}\left(\frac{\partial u_{r 1}}{\partial \theta} \sin \theta\right)\right] \\
\frac{\partial u_{\theta 1}}{\partial t} & =-\frac{1}{r} \frac{\partial p_{1}}{\partial \theta}+ \\
& +O h\left[\frac{1}{r^{2}} \frac{\partial}{\partial r}\left(r^{2} \frac{\partial u_{\theta 1}}{\partial r}\right)+\frac{1}{r^{2}} \frac{\partial}{\partial \theta}\left(\frac{1}{\sin \theta} \frac{\partial}{\partial \theta}\left(u_{\theta 1} \sin \theta\right)\right)+\frac{2}{r^{2}} \frac{\partial u_{r 1}}{\partial \theta}\right]
\end{aligned}
$$

For the boundary conditions of first order to be satisfied at $r=1$ we obtain

$$
\begin{array}{rlrl}
u_{r 1} & =\frac{\partial \eta_{1}}{\partial t} & \text { kinematic } \\
r \frac{\partial}{\partial r}\left(\frac{u_{\theta 1}}{r}\right)+\frac{1}{r} \frac{\partial u_{r 1}}{\partial \theta} & =0 & \text { zero shear stress } \\
-p_{1}+2 O h \frac{\partial u_{r 1}}{\partial r}-\left(2 \eta_{1}+\frac{\partial \eta_{1}}{\partial \theta} \cot \theta+\frac{\partial^{2} \eta_{1}}{\partial \theta^{2}}\right) & =0 & & \text { zero normal stress }
\end{array}
$$

Furthermore, the initial conditions of first order are

$$
\eta_{1}(\theta, 0)=P_{m}(\cos \theta) \text { and } \frac{\partial \eta_{1}}{\partial t}(\theta, 0)=0
$$

The first initial condition determines the initial shape of the deformed drop by a Legendre polynomial $P_{m}$ of order $m$.

\section{Second-order equations}

The second-order equations are obtained as all the terms in the above expansions with the parameter $\eta_{0}$ squared. The second-order continuity and momentum equations read

$$
\begin{gathered}
\frac{1}{r^{2}} \frac{\partial}{\partial r}\left(r^{2} u_{r 2}\right)+\frac{1}{r \sin \theta} \frac{\partial}{\partial \theta}\left(u_{\theta 2} \sin \theta\right)=0 \\
\frac{\partial u_{r 2}}{\partial t}-O h\left[\frac{1}{r^{2}} \frac{\partial^{2}}{\partial r^{2}}\left(r^{2} u_{r 2}\right)+\frac{1}{r^{2} \sin \theta} \frac{\partial}{\partial \theta}\left(\frac{\partial u_{r 2}}{\partial \theta} \sin \theta\right)\right]+\frac{\partial p_{2}}{\partial r}=\frac{u_{\theta 1}^{2}}{r}-u_{r 1} \frac{\partial u_{r 1}}{\partial r}-\frac{u_{\theta 1}}{r} \frac{\partial u_{r 1}}{\partial \theta}
\end{gathered}
$$




$$
\begin{aligned}
\frac{\partial u_{\theta 2}}{\partial t} & -O h\left[\frac{1}{r^{2}} \frac{\partial}{\partial r}\left(r^{2} \frac{\partial u_{\theta 2}}{\partial r}\right)+\frac{1}{r^{2}} \frac{\partial}{\partial \theta}\left(\frac{1}{\sin \theta} \frac{\partial}{\partial \theta}\left(u_{\theta 2} \sin \theta\right)\right)\right. \\
& \left.+\frac{2}{r^{2}} \frac{\partial u_{r 2}}{\partial \theta}\right]+\frac{1}{r} \frac{\partial p_{2}}{\partial \theta}=-u_{r 1} \frac{\partial u_{\theta 1}}{\partial r}-\frac{u_{\theta 1}}{r} \frac{\partial u_{\theta 1}}{\partial \theta}-\frac{u_{r 1} u_{\theta 1}}{r}
\end{aligned}
$$

The boundary conditions of second order for the kinematics, and for zero shear and normal stresses, at $r=1$ are

$$
\begin{aligned}
u_{r 2}-\frac{\partial \eta_{2}}{\partial t} & =\frac{u_{\theta 1}}{r} \frac{\partial \eta_{1}}{\partial \theta}-\eta_{1} \frac{\partial u_{r 1}}{\partial r} \\
r \frac{\partial}{\partial r}\left(\frac{u_{\theta 2}}{r}\right)+\frac{1}{r} \frac{\partial u_{r 2}}{\partial \theta} & =-\eta_{1} \frac{\partial}{\partial r}\left(r \frac{\partial}{\partial r}\left(\frac{u_{\theta 1}}{r}\right)+\frac{1}{r} \frac{\partial u_{r 1}}{\partial \theta}\right) \\
-2\left(r \frac{\partial}{\partial r}\left(\frac{u_{r 1}}{r}\right)-\frac{1}{r} \frac{\partial u_{\theta 1}}{\partial \theta}\right) \frac{1}{r} \frac{\partial \eta_{1}}{\partial \theta} & \\
-p_{2}+2 O h \frac{\partial u_{r 2}}{\partial r}-\left(2 \eta_{2}+\frac{\partial \eta_{2}}{\partial \theta} \cot \theta+\frac{\partial^{2} \eta_{2}}{\partial \theta^{2}}\right) & =\eta_{1} \frac{\partial p_{1}}{\partial r} \\
-2 O h\left[\eta_{1} \frac{\partial^{2} u_{r 1}}{\partial r^{2}}-\frac{1}{r} \frac{\partial \eta_{1}}{\partial \theta}\left(r \frac{\partial}{\partial r}\left(\frac{u_{\theta 1}}{r}\right)+\frac{1}{r} \frac{\partial u_{r 1}}{\partial \theta}\right)\right] & -\left(2 \eta_{1}^{2}+2 \eta_{1} \frac{\partial \eta_{1}}{\partial \theta} \cot \theta+2 \eta_{1} \frac{\partial^{2} \eta_{1}}{\partial \theta^{2}}\right)
\end{aligned}
$$

Furthermore, the initial conditions of second order are

$$
\eta_{2}(\theta, 0)=-\frac{1}{2 m+1} \quad \text { and } \quad \frac{\partial \eta_{2}}{\partial t}(\theta, 0)=0
$$

The following section presents solutions to these sets of first- and second-order equations.

\section{Solutions}

\section{First-order solutions}

The first-order equations describe the linear problem. The two-dimensional flow field allows the method of the Stokesian stream function to be applied for determining the velocity field. The streamfunction $\psi(r, \theta, t)$ is defined by its relations to the velocity components $u_{r 1}$ and $u_{\theta 1}$ [8]

$$
u_{r 1}=-\frac{1}{r^{2} \sin \theta} \frac{\partial \psi}{\partial \theta} \quad \text { and } \quad u_{\theta 1}=\frac{1}{r \sin \theta} \frac{\partial \psi}{\partial r}
$$

The first-order drop surface deformation is governed by the Legendre polynomial of the initial deformation. The solution is therefore sought in the form

$$
\eta_{1}=\hat{\eta}_{1} P_{m}(\cos \theta) e^{-\alpha_{m} t}
$$

with the first-order initial surface amplitude $\hat{\eta}_{1}$ and the complex angular frequency $\alpha_{m}$ of the drop for mode $m$.

Eliminating pressure from the first-order momentum equation in a vectorial form yields the differential equation for the stream function

$$
\left(\frac{1}{O h} \frac{\partial}{\partial t}-E_{s}^{2}\right)\left(E_{s}^{2} \psi\right)=0 \quad \text { with the operator [8] } \quad E_{s}^{2}=\frac{\partial^{2}}{\partial r^{2}}+\frac{\sin \theta}{r^{2}} \frac{\partial}{\partial \theta}\left(\frac{1}{\sin \theta} \frac{\partial}{\partial \theta}\right) \text {. }
$$

The solution may be represented as the sum $\psi=\psi_{1}+\psi_{2}$, with [9]

$$
\psi_{1} \propto r^{m+1} \sin ^{2} \theta P_{m}^{\prime}(\cos \theta) e^{-\alpha_{m} t} \quad \text { and } \quad \psi_{2} \propto q r j_{m}(q r) \sin ^{2} \theta P_{m}^{\prime}(\cos \theta) e^{-\alpha_{m} t}
$$

where $P_{m}^{\prime}(\cos \theta)$ is the first-order derivative of the Legendre polynomial $P_{m}$ with respect to its argument and $j_{m}(q r)$ the spherical Bessel function of the first kind and order $m$, with $q=$ $\sqrt{\alpha_{m} / O h}$. The radial and angular first-order velocity components are obtained as

$$
u_{r 1}=-\left[C_{1 m} r^{m-1}+C_{2 m} q^{2} \frac{j_{m}(q r)}{q r}\right] m(m+1) P_{m}(\cos \theta) e^{-\alpha_{m} t}
$$



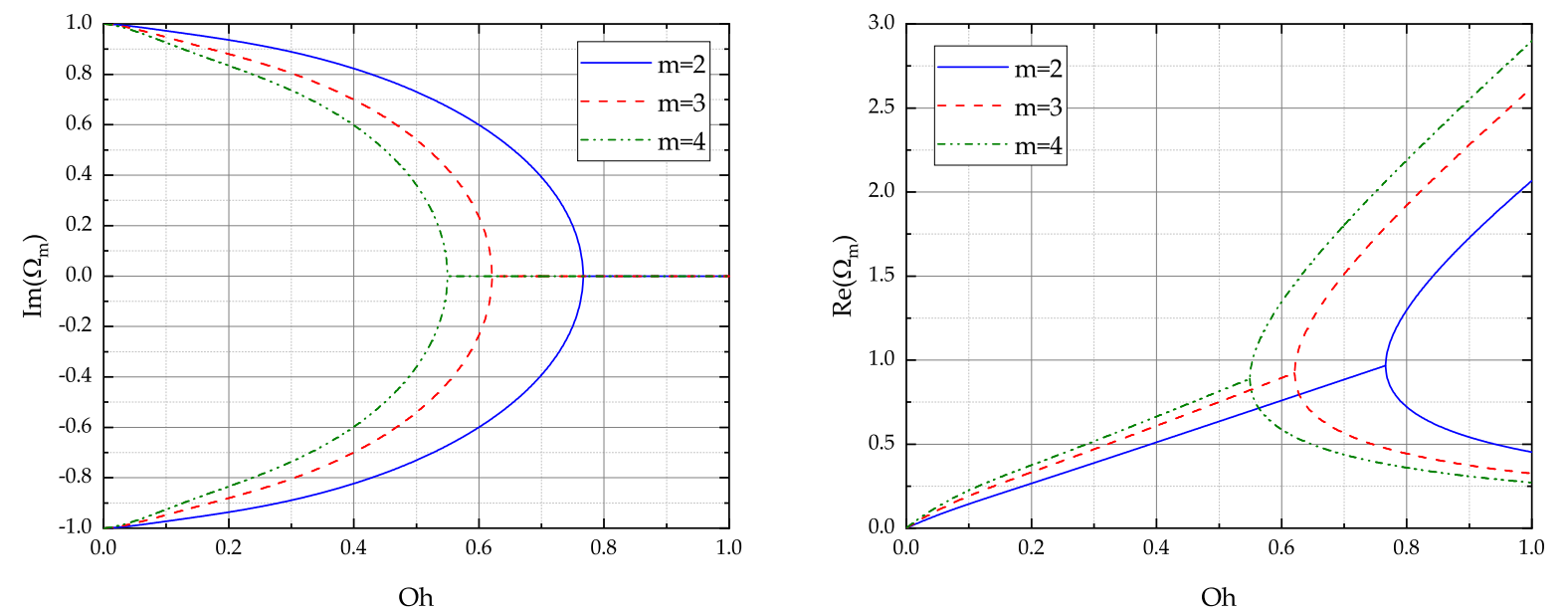

Figure 1. Solutions of the characteristic equation for varying Ohnesorge number. Left - imaginary and right - real part of the nondimensional frequency. The critical Ohnesorge number for mode $m=2$ has the value 0.7665 [5].

and

$$
u_{\theta 1}=\left[C_{1 m}(m+1) r^{m-1}+C_{2 m} q^{2}\left((m+1) \frac{j_{m}(q r)}{q r}-j_{m+1}(q r)\right)\right] \sin \theta P_{m}^{\prime}(\cos \theta) e^{-\alpha_{m} t},
$$

respectively. The two integration constants $C_{1 m}$ and $C_{2 m}$ are determined by the first-order kinematic and zero shear stress boundary conditions. The first-order pressure field $p_{1}$ is obtained by integration of one component of the momentum equation as

$$
p_{1}=-C_{1 m}(m+1) \alpha_{m} r^{m} P_{m}(\cos \theta) e^{-\alpha_{m} t} \text {. }
$$

The characteristic equation for the complex angular frequency $\alpha_{m}$ is derived from (24) as

$$
\frac{\alpha_{m, 0}^{2}}{\alpha_{m}^{2}}=\frac{2\left(m^{2}-1\right)}{q^{2}-2 q j_{m+1} / j_{m}}-1+\frac{2 m(m-1)}{q^{2}}\left[1+\frac{2(m+1) j_{m+1} / j_{m}}{2 j_{m+1} / j_{m}-q}\right]
$$

with $\alpha_{m, 0}=[m(m-1)(m+2)]^{1 / 2}$. The spherical Bessel functions are taken at the value $q$ of their arguments. The equation is identical to the results in [2] and [10].

The characteristic equation exhibits pairs of complex conjugate solutions, as depicted in Fig. 1 for the first three deformation modes $m=2,3,4$. For Ohnesorge numbers below the critical value (of 0.7665 for mode $m=2$ [5]), the drop performs damped shape oscillations. For greater Ohnesorge numbers, the drop motion is aperiodic.

The existence of pairs of solutions of the characteristic equation requires representation in the first-order solutions. We therefore formulate the first-order deformation of the drop surface as

$$
\eta_{1}(\theta, t)=\left(\hat{\eta}_{1}^{(p)} e^{-\alpha_{m}^{(p)} t}+\hat{\eta}_{1}^{(n)} e^{-\alpha_{m}^{(n)} t}\right) P_{m}(\cos \theta)
$$

The first-order initial conditions (18) require that initially the drop surface is governed by the function $P_{m}(\cos \theta)$ and is at rest. For the amplitudes $\hat{\eta}_{1}^{(p)}$ and $\hat{\eta}_{1}^{(n)}$, these conditions reveal

$$
\hat{\eta}_{1}^{(p)}=-\frac{\alpha_{m}^{(n)}}{\alpha_{m}^{(p)}-\alpha_{m}^{(n)}} \quad \text { and } \quad \hat{\eta}_{1}^{(n)}=\frac{\alpha_{m}^{(p)}}{\alpha_{m}^{(p)}-\alpha_{m}^{(n)}}
$$

where we denote the solutions with positive and negative imaginary parts by superscripts $(p)$ and $(n)$, respectively. The first-order radial velocity component $u_{r 1}$, as an example, reads

$$
\begin{gathered}
u_{r 1}(r, \theta, t)=-\hat{\eta}_{1}^{(p)} \alpha_{m}^{(p)}\left[r^{m-1}+\frac{2\left(m^{2}-1\right)}{2 q^{(p)} Q_{m}^{(p)}-q^{(p)^{2}}}\left(r^{m-1}-\frac{j_{m}\left(q^{(p)} r\right)}{j_{m}\left(q^{(p)}\right)} \frac{1}{r}\right)\right] P_{m}(\cos \theta) e^{-\alpha_{m}^{(p)} t} \\
-\hat{\eta}_{1}^{(n)} \alpha_{m}^{(n)}\left[r^{m-1}+\frac{2\left(m^{2}-1\right)}{2 q^{(n)} Q_{m}^{(n)}-q^{(n)^{2}}}\left(r^{m-1}-\frac{j_{m}\left(q^{(n)} r\right)}{j_{m}\left(q^{(n)}\right)} \frac{1}{r}\right)\right] P_{m}(\cos \theta) e^{-\alpha_{m}^{(n)} t}
\end{gathered}
$$


with the definition of $Q_{m}=j_{m+1}(q) / j_{m}(q)$ with $q^{(p)}$ and $q^{(n)}$ for $\alpha_{m}^{(p)}$ and $\alpha_{m}^{(n)}$.

\section{Second-order solutions}

We derive the second-order solutions from pressure. The second-order pressure is sought as

$$
p_{2}(r, \theta, t)=p_{21}(r, \theta, t)+p_{22}(r, \theta, t)
$$

where subscript 21 indicates the solution of the second-order equations system including the first-order term products, and subscript 22 the solutions of the homogeneous system. The length restrictions to this conference contribution make a full presentation of the second-order solutions impossible.

The solutions with subscripts 21 are first determined. Eliminating the second-order velocity from the second-order equations of motion (19)-(22), we obtain the differential equation for the second-order pressure $p_{21}$

$$
\begin{aligned}
\frac{1}{r^{2}} \frac{\partial}{\partial r}\left(r^{2} \frac{\partial p_{21}}{\partial r}\right) & +\frac{1}{r \sin \theta} \frac{\partial}{\partial \theta}\left(\frac{\sin \theta}{r} \frac{\partial p_{21}}{\partial \theta}\right)=-\frac{1}{r^{2}} \frac{\partial}{\partial r}\left[r^{2}\left(u_{r 1} \frac{\partial u_{r 1}}{\partial r}+\frac{u_{\theta 1}}{r} \frac{\partial u_{r 1}}{\partial \theta}-\frac{u_{\theta 1}^{2}}{r}\right)\right] \\
& -\frac{1}{r \sin \theta} \frac{\partial}{\partial \theta}\left[\left(u_{r 1} \frac{\partial u_{\theta 1}}{\partial r}+\frac{u_{\theta 1}}{r} \frac{\partial u_{\theta 1}}{\partial \theta}+\frac{u_{r 1} u_{\theta 1}}{r}\right) \sin \theta\right]
\end{aligned}
$$

which we re-formulate using the Lamé identity and the properties of the present first-order velocity field into the form

$$
\Delta\left[p_{21}+\vec{v}_{1}^{2} / 2\right]=\frac{1}{O h} \operatorname{div}\left[\frac{1}{r^{2} \sin ^{2} \theta} \frac{\partial \psi_{2}}{\partial t} \vec{\nabla} \psi\right]
$$

This is a Poisson equation for the modified pressure $\mathcal{P}_{21}=p_{21}+\vec{v}_{1}^{2} / 2$, found in the cylindrical formulation for a jet in [11]. The structure of the solution in terms of its dependency on the polar angular coordinate and on time is determined by $\vec{v}_{1}^{2}$, and by the first-order term products on the right-hand side of equation (39).

In solving the Poisson equation, the general solution of the homogeneous (i.e., the Laplace) equation, for the time dependency according to $\exp \left(-2 \alpha_{m}^{(p)} t\right)$, reads

$$
\mathcal{P}_{21, H}=-\sum_{l=0}^{\infty} D_{21 l^{(p)}} r^{l} P_{l}(\cos \theta) e^{-2 \alpha_{m}^{(p)} t}
$$

The particular solution of the Poisson equation is found representing products of Legendre functions on the right-hand side of the equation by series expansions of Legendre polynomials. Details of the derivation of those solutions will be presented elsewhere [12]. From the pressure field $p_{21}$ and the equations of motion (19)-(22), the corresponding second-order velocities $u_{r 2}$ and $u_{\theta 2}$ are determined. It follows the determination of the 21 contributions to the surface deformation $\eta_{21}$ corresponding to the three behaviours in time. Due to space limits, the details will be presented elsewhere [12].

The second contributions to the second-order solutions, with subscript 22, are determined from the homogeneous forms of the equations (19)-(22). This set of equations has the same structure as at first order, where the complex angular frequency $\alpha_{m}$ is replaced by a different $\alpha_{2 k}$, and the deformation amplitude of the drop surface $\hat{\eta}_{1}$ by $\hat{\eta}_{22 k}$. The general solution of the Laplace equation is given by a series expansion in all the eigen-solutions with subscript $k$. The new complex angular frequency $\alpha_{2 k}$ is obtained as a solution of the characteristic equation (33), but with the mode $m$ replaced by the summation index $k$.

\section{Results and discussion}

This section presents the weakly non-linear analysis results for initial modes of deformation $m=2,3$ and 4 . The analysis, however, allows for modes $m>4$ also. We first quantify the 

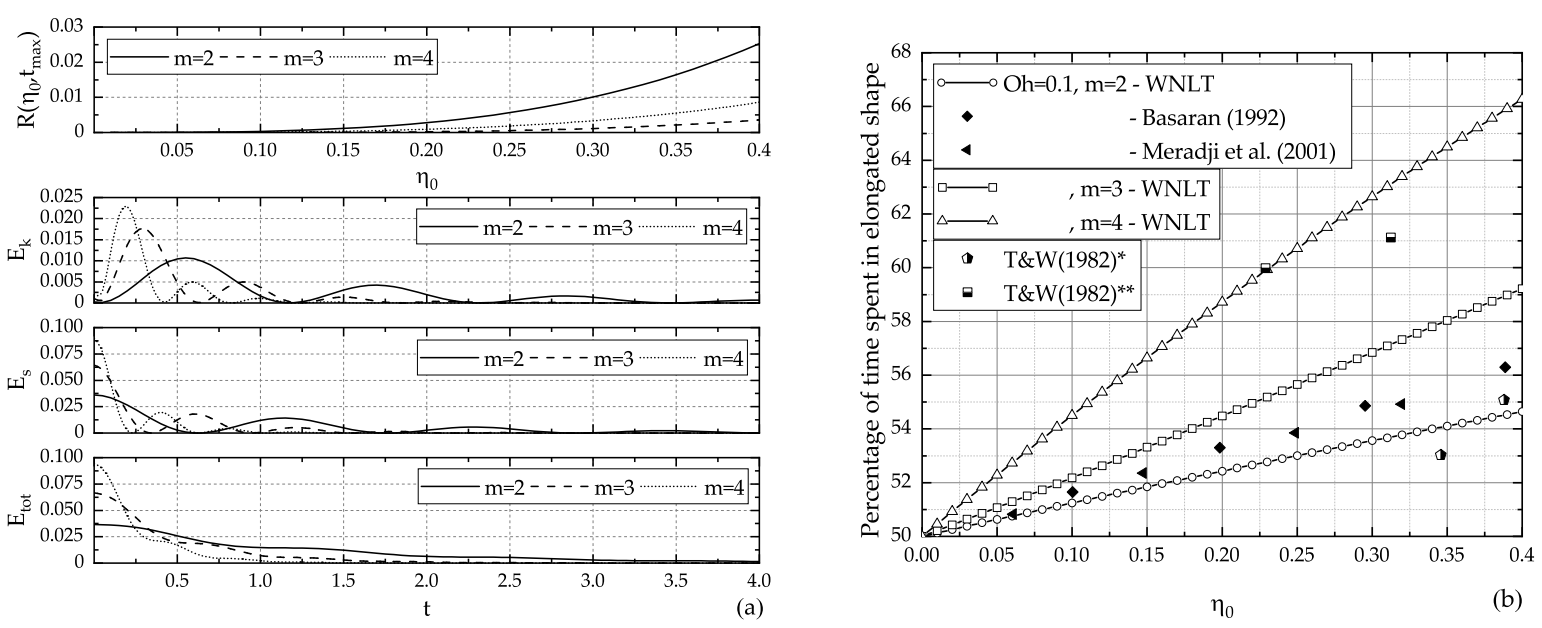

Figure 2. (a) Maximum relative drop volume deviation as a function of the deformation parameter $\eta_{0}$; kinetic, surface and total energies as functions of time for $O h=0.1, \eta_{0}=0.3$. (b) Percentage of the drop oscillation period spent in a shape elongated along the symmetry axis, as a function of the deformation parameter. T\&W(1982)* denotes experimental data by [13] for initial steady prolate drive, and $T \& W(1982)^{\star \star}$ for initial static prolate shape.

conservation of the drop volume as a function of deformation amplitude and time. The nondimensional drop volume equals $V(t)=(2 \pi / 3) \int_{-1}^{1} r_{s}^{3}(x, t) d x$. The relative volume deviation from the exact value $V_{s}=4 \pi / 3$ is

$$
\left(V(t)-V_{s}\right) / V_{s}=: R\left(\eta_{0}, t\right)=\frac{1}{2} \int_{-1}^{1}\left[r_{s}^{3}(x, t)-1\right] d x,
$$

where $x=\cos \theta$. The values of $R$ are positive, oscillate in time and increase with $\eta_{0}$. The top diagram in Fig. 2 (a) presents the relative volume deviation for the modes of initial deformation $m=2,3$ and 4 at the instant of time $t=t_{\max }$ of its largest value. For a given mode of initial deformation, the relative volume deviation increases with the deformation parameter $\eta_{0}$. For $\eta_{0}=0.4$ and $m=2,3$ and $4, R$ at $t_{\max }$ is around $2.5 \%, 0.4 \%$ and $0.8 \%$, respectively.

Calculation of the energy contents of the drop gives insight into the transformation of the mechanical energy forms during the oscillation and shows the dissipation of mechanical energy. These predictions are relevant for the transport processes across the drop surface. The kinetic $E_{k}$, surface $E_{s}$ and total energies $E_{t o t}$ for the example of $O h=0.1$ are shown in Fig. 2 (a). The values in the diagrams are non-dimensional with the surface energy $4 \pi a^{2} \sigma$. Corresponding to the truncated series expansions, the calculation of the energies is for second order. At the initial time, the droplet is the most deformed and, consequently, contains the maximum surface energy. The initial conditions ensure quiescent drop surface. The bulk of the drop, however, is in motion. The sum of the kinetic and surface energies, which are continuously transformed into each other, reveals the total energy as a function of time. Due to dissipation, the latter energy decreases monotonically in time, as expected. The rate of decrease is greatest when the rate of dissipation is highest, and at the beginning of the motion it is largest for the highest deformation mode studied.

One well-known non-linear effect in drop oscillations with $m=2$ is an asymmetry of the times the drop spends in the oblate and prolate deformed states. One expects this effect to be less pronounced in the present viscous than in the inviscid case. The second-order solutions can represent this phenomenon. In Fig. 2 (b), the solutions from the weakly non-linear theory (WNLT) for $m=2,3$ and 4 show the time the drop spends in the elongated form, determined from the first oscillation. The relatively high Ohnesorge number of 0.1 yields only a few oscillations, where the second and following periods exhibit very small amplitudes and thus do not show non-linearities. The numerical results by [14] and [15] also show the first oscillation 
only, but start the simulations from the drop bulk at rest. Our results for time asymmetry of the $m=2$ oscillations agree very well with the experimental work by [13] who investigated largeamplitude oscillations for different initial states. Since WNLT includes an initial bulk drop motion, it matches the experimental results for oscillations starting from the steady prolate drive state $\left(T \& W(1982)^{*}\right)$. The excess time increases with increasing initial deformation mode number.

\section{Conclusions}

A weakly non-linear analysis of shape oscillations of a Newtonian, axisymmetric liquid drop in a vacuum was performed. The volume deviation during the oscillations depends on the deformation parameter and on the mode of initial deformation. Deviations are below $2.5 \%$ of the spherical volume in all cases investigate. The second-order solutions represent the time asymmetry in the oscillations, revealing the excess time spent in an elongated drop form for different initial modes. For $m=2$ and initially driven oscillations, the results are in good agreement with experimental literature [13]. The present results reveal the exchange between the kinetic and surface energies, and the dissipation of total energy in time, where dissipation rates are highest when velocities are largest.

\section{Acknowledgements}

Financial support of this research project by the Austrian Science Fund (FWF) through project number I3326-N32 in the DACH framework is gratefully acknowledged.

\section{References}

[1] Rayleigh, J.W.S., 1879, Proc. R. Soc. London A, 29, pp. 71-97.

[2] Lamb, H., 1881, Proc. London Math. Soc., 13, pp. 51-66.

[3] Lamb, H., 1932, Hydrodynamics, 6th edn. Cambridge.

[4] Miller, C.A. and Scriven, L.E., 1968, J. Fluid Mech., 32, pp. 417-435.

[5] Prosperetti, A., 1980, J. Fluid Mech., 100, pp. 333-347.

[6] Tsamopoulos, J.A. and Brown, R.A., 1983, J. Fluid Mech., 127, pp. 519-537.

[7] Zrnić, D., Brenn, G., 2021a, J. Fluid Mech., under review.

[8] Bird, R.B., Stewart, W.E., Lightfoot, E.N., 1962, Transport Phenomena, John Wiley \& Sons, New York.

[9] Tomotika, S., 1935, Proc. R. Soc. London A, 150, pp. 322-337.

[10] Chandrasekhar, S., 1959, Proc. London Math. Soc., 9, pp. 141-149.

[11] Renoult, M.-C., Brenn, G., Plohl, G., Mutabazi, I., 2018, J. Fluid Mech., 856, pp. 169-201.

[12] Zrnić, D., Brenn, G., 2021b, J. Fluid Mech., manuscript in preparation.

[13] Trinh, E. H., and Wang, T.G., 1982, In Proc. 2nd Int. Colloq. on Drops and Bubbles. JPL Publ., Pasadena (CA, USA), pp. 82-87.

[14] Meradji, S., Lyubimova T. P., Lyubimov, D.V., Roux, B., 2001, Cryst. Res. Technol., 36, pp. 729-744.

[15] Basaran, O.A., 1993, J. Fluid Mech., 241, pp. 169-198. 